ACUTE CORONARY SYNDROMES

\title{
Coronary artery flow velocity profile measured by transthoracic Doppler echocardiography predicts myocardial viability after acute myocardial infarction
}

\author{
Antti Saraste, Juha W Koskenvuo, Markku Saraste, Jussi Pärkkä, Jyri Toikka, Alexandru Naum, \\ Heikki Ukkonen, Juhani Knuuti, Juhani Airaksinen, Jaakko Hartiala
}

Heart 2007;93:456-457. doi: 10.1136/hrt.2006.094995

See end of article for authors' affiliations

Correspondence to:

Dr A Saraste, Department of Clinical Physiology and

Nuclear Medicine, Turku

University Hospital,

Kiinamyllynkatu 6-8, 20520

Turku, Finland;

antti.saraste@utu.fi

Accepted

26 September 2006

Published Online First

28 November 2006

\begin{abstract}
Objective: To study whether flow velocity profile in the left anterior descending coronary artery (LAD) measured by transthoracic Doppler echocardiography (TDE) predicts myocardial viability after reperfused anterior acute myocardial infarction (AMI).

Patients and methods: 15 patients who had their first anterior ST elevation AMI and were successfully reperfused by coronary angioplasty and five controls without coronary artery disease were selected. Blood flow velocity spectrum was measured from the mid-LAD by TTDE 3 days after coronary angioplasty. Myocardial viability in the LAD region was quantified 3 months after AMI by relative uptake of 18Ffluorodeoxyglucose (FDG) imaged with positron emission tomography. Myocardium was graded as viable, partially viable or non-viable (relative FDG uptake $>85 \%, 67-85 \%$ and $<67 \%$, respectively). Main outcome measures were diastolic deceleration time (DDT) of LAD flow velocity 3 days after AMI and myocardial viability 3 months after AMI.

Results: DDT of LAD flow velocity correlated with myocardial FDG uptake in the LAD region $(r=0.91$, $\mathrm{p}<0.01)$. DDT was markedly longer in patients with viable myocardium $(876 \pm 76 \mathrm{~ms}, \mathrm{n}=3$ ) than partially viable $(356 \pm 89 \mathrm{~ms}, \mathrm{n}=6, \mathrm{p}<0.01)$, or non-viable myocardium $(128 \pm 13 \mathrm{~ms}, \mathrm{n}=6, \mathrm{p}<0.01)$. In controls, DDT was comparable $(909 \pm 76 \mathrm{~ms}, \mathrm{n}=5)$ to patients with viable myocardium. DDT $<190 \mathrm{~ms}$ was always associated with non-viable myocardium.

Conclusions: DDT of LAD flow velocity is strongly associated with myocardial viability after reperfused anterior AMI. Non-invasive TTDE of the LAD may be used in the acute phase to predict long-term viability of the jeopardised myocardium.
\end{abstract}

T he no-reflow phenomenon in acute myocardial infarction (AMI) is associated with advanced microvascular damage and characteristic flow velocity profile in intracoronary flow measurements. ${ }^{1}$ A recent study suggested that short deceleration time of early-diastolic flow velocity (diastolic deceleration time, DDT) in the left anterior descending coronary artery (LAD) recorded with non-invasive transthoracic Doppler echocardiography (TTDE) identifies the no-reflow phenomenon after anterior AMI. ${ }^{2}$ However, whether flow velocity profile assessed by TTDE predicts myocardial viability after AMI remains uncertain. ${ }^{2-5}$ Evaluation of myocardial glucose utilisation with 18F-fluorodeoxyglucose (FDG) and positron emission tomography (PET) is considered as the most reliable tool to assess myocardial viability. Thus, we decided to study whether LAD flow velocity profile measured by TTDE predicts myocardial viability as assessed by PET after anterior AMI.

\section{PATIENTS AND METHODS}

The study group consisted of 15 patients aged 59 (13) years (12 males) who had their first anterior ST elevation AMI and successful angioplasty of LAD performed (revascularisation delay 7 (11) h) in the Turku University Hospital, Turku, Finland. The control group consisted of five males aged 60 (2) years without coronary artery disease. Ethics committee of the University of Turku and National Agency for Medicines in Finland approved the study protocol and the patients gave written informed consent.

Blood flow velocity profile in the mid-LAD was recorded by TTDE (Acuson Sequoia C512 with $3.5 \mathrm{MHz}$ transducer;
Siemens Acuson, Mountain View, California, USA) in the acute phase 3 (0.5) days after AMI and again at the time of PET study. We measured DDT in at least three cardiac cycles (intraobserver and interobserver variations in repeated measurements were $6 \%$ and $9 \%$, respectively). Average wall motion score in the LAD region and global ejection fraction (EF) by Simpson's biplane method were also assessed

Myocardial viability in the LAD region was assessed by relative uptake of FDG imaged with PET (eight-ring ECAT 931/08-12 tomograph, CTI/Siemens, Knoxville, Tennessee, USA) 3 (0.8) months after AMI. The uptake of FDG was normalised to a segment in the non-infarcted myocardium that was supplied by a nonstenosed coronary artery and showed the highest tracer uptake. Myocardium was graded as viable, partially viable or non-viable (relative FDG uptake $>85 \%, 67-85 \%$ and $<67 \%$, respectively).

Data are expressed as mean (SD). Pearson's correlation coefficients (continuous variables), Student's t test (two groups), one-way ANOVA and Bonferroni's method (multiple groups), $\chi^{2}$ test (categorical data) and receiver operator characteristic curves were used in the analysis of data, $\mathrm{p}<0.05$ was considered statistically significant.

\section{RESULTS}

Age, sex, risk factors for coronary artery disease, revascularisation delay, TIMI flow grade after angioplasty and the presence

Abbreviations: AMI, acute myocardial infarction; DDT, diastolic deceleration time; EF, ejection fraction; FDG, fluorodeoxyglucose; LAD, left anterior descending coronary artery; PET, positron emission tomography; TTDE, transthoracic Doppler echocardiography 
of collaterals in angiography were comparable between patients who had non-viable $(n=6)$, partially viable $(n=6)$ or viable $(\mathrm{n}=3)$ myocardium. Although EF was comparable in the acute phase $(44 \% \quad(6 \%), 46 \% \quad(8 \%)$ and $51 \% \quad(5 \%)$ respectively, $\mathrm{p}=0.16)$, it was lower in patients who had non-viable than partially viable or viable myocardium 3 months later $(39 \%$ $(11 \%), 51 \%(9 \%)$, and $60 \%(3 \%)$ respectively; $p=0.02)$.

Myocardial FDG uptake in the LAD region correlated with DDT of LAD flow velocity measured 3 days after AMI $(r=0.91$, $\mathrm{p}<0.01$; fig 1). Patients who had non-viable myocardium showed markedly shorter DDT (128 (13) ms) than patients with partially viable (356 (89) $\mathrm{ms}$ ) or viable myocardium (876 (76) $\mathrm{ms}$ ) in the acute phase (fig 1). In controls, DDT (909 (76) ms) was comparable to patients with viable myocardium. Using $190 \mathrm{~ms}$ as the optimal cut-off point DDT always correctly identified nonviable myocardium (sensitivity and specificity 100\%).

There was also a correlation between DDT in the acute phase and EF 3 months after AMI $(\mathrm{r}=0.59, \mathrm{p}<0.05)$ and change in EF from the acute phase to 3 months after AMI $(r=0.64, p=0.01)$. Average DDT in the acute phase was also shorter in patients with non-functional myocardium in the LAD region (average wallmotion score $>2$ ) after 3 months than in the other patients ( 162 (91) vs $550(283) \mathrm{ms}, \mathrm{p}<0.01)$. Using $190 \mathrm{~ms}$ as the optimal cutoff point, DDT identified non-functional myocardium with $86 \%$ sensitivity and $100 \%$ specificity.

Compared with the acute phase, DDT prolonged in patients with non-viable myocardium 3 months after AMI (234
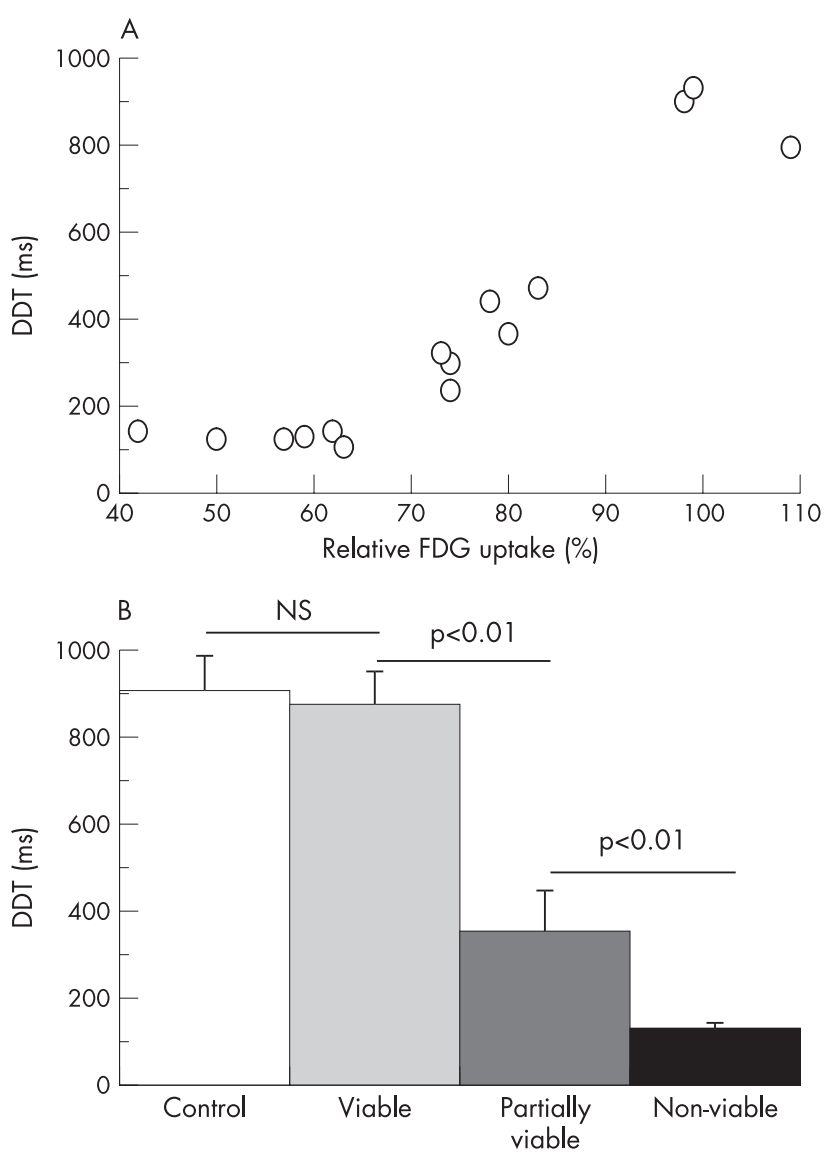

Figure 1 There is a correlation between diastolic flow velocity of left anterior descending coronary artery (LAD) flow velocity in the acute phase and relative fluorodeoxygluocose-uptake in the LAD region 3 months after acute myocardial infarction $(A, r=0.91, p<0.01)$. Controls $(n=5)$ and patients with viable myocardium $(n=6)$ had longer diastolic deceleration time (DDT) than patients with partially viable $(n=6)$ or non-viable myocardium $(B, n=6)$. FDG, fluorodeoxyglucose; NS, not significant.
(26) $\mathrm{ms}, \mathrm{p}=0.01$ ), but remained constant in patients with partially viable (492 (87) ms) and viable (970 (192) ms) myocardium. After 3 months of AMI, uptake of FDG and DDT correlated $(\mathrm{r}=0.76, \mathrm{p}<0.01)$ and $390 \mathrm{~ms}$ was the optimal cutoff point for identifying non-viable myocardium (sensitivity $100 \%$ and specificity $88 \%$ ).

\section{DISCUSSION}

Our results provide evidence that TTDE of the LAD may be used to predict viability of the jeopardised myocardium after AMI. There was a strong correlation between DDT of LAD flow velocity in the acute phase and the amount of viable myocardium as assessed by FDG PET 3 months after AMI.

Previously, an association between DDT and recovery of regional left ventricular function after anterior AMI has been found in two TTDE studies. ${ }^{2}{ }^{3}$ In two other studies, however, no such association was found. ${ }^{45}$ Several factors may contribute to these diverging results as relatively small numbers of patients have been studied using varying methodology. One is that the flow velocity profiles may vary between middle and distal parts of LAD. The former has been used in intracoronary measurements, ${ }^{1}$ whereas the latter was apparently used in TTDE studies. ${ }^{45}$ Another is that, categorisation of myocardium as functional or non-functional by echocardiography without pharmacological provocation may not be an accurate indicator of viability, since ischaemia-reperfusion injury often results in a mixture of scar and viable myocytes.

The strength of our study is that we assessed myocardial viability by FDG uptake imaged with PET, which is considered as the most reliable tool for this purpose. Its quantitative nature allows assessment of the amount of viable tissue as a continuum from fully viable, through partially viable in the areas of partial infarction, to non-viable scar. Moreover, we measured DDT 3 days after AMI, which best-predicted the functional outcome in serial measurements. ${ }^{3}$ Our study, being limited by the small number of patients, warrants larger studies to explore the value of coronary artery flow velocity profile in predicting long-term outcomes after AMI.

\section{Authors' affiliations}

Juha W Koskenvuo, Markku Saraste, Jussi Pärkkä, Jyri Toikka, Jaakko Hartiala, Department of Clinical Physiology and Nuclear Medicine, Turku University Hospital, Turku, Finland

Antti Saraste, Heikki Ukkonen, Juhani Airaksinen, Department of

Medicine, Turku University Hospital, Turku, Finland

Alexandru Naum, Heikki Ukkonen, Juhani Knuuti, Turku PET centre,

Turku, Finland

Funding: The study has been financially supported by clinical research funds of the Turku University Hospital, Turku, Finland.

Competing interests: None.

\section{REFERENCES}

1 Iwakura K, Ito $\mathrm{H}$, Takiuchi S, et al. Alteration in the coronary blood flow velocity pattern in patients with no reflow and reperfused acute myocardial infarction. Circulation 1996:94:1269-75.

2 Iwakura K, Ito H, Kawano S, et al. Assessing myocardial perfusion with the transthoracic Doppler technique in patients with reperfused anterior myocardial infarction: comparison with angiographic, enzymatic and electrocardiographic indices. Eur Heart J 2004;25:1526-33.

3 Hozumi T, Kanzaki Y, Ueda Y, et al. Coronary flow velocity analysis during short term follow up after coronary reperfusion: use of transthoracic Doppler echocardiography to predict regional wall motion recovery in patients with acute myocardial infarction. Heart 2003;89:1163-68.

4 Voci $\mathbf{P}$, Mariano $E$, Pizzuto $F$, et al. Coronary recanalization in anterior myocardial infarction. The open perforator hypothesis. J Am Coll Cardiol 2002;40:1205-13

5 Nohtomi Y, Takeuchi M, Nagasawa K, et al. Persistence of systolic coronary flow reversal predicts irreversible dysfunction after reperfused anterior myocardial infarction. Heart 2003;89:382-8. 